\title{
Spacing and the transition from calculation to retrieval
}

\author{
Timothy C. Rickard, Jonas Sin-Heng Lau, and Harold Pashler \\ University of California, San Diego, La Jolla, California
}

\begin{abstract}
Many arithmetic problems can be solved in two ways - by a calculation involving several steps and by direct retrieval of the answer. With practice on particular problems, memory retrieval tends to supplant calculationan important aspect of skill learning. We asked how the distribution of practice on particular problems affects this kind of learning. In two experiments, subjects repeatedly worked through sets of multiple-digit multiplication problems. The size of the trained problem set was varied. Using a smaller set size (with shorter average time between problem repetitions) showed faster responses and an earlier transition to retrieval during training. However, in a test session presented days later, the pattern reversed, with faster responses and more retrieval for the large set size. Evidently, maximizing the occurrence of direct retrieval within training is not the best way to promote learning to retrieve the answer. Practical implications are discussed.
\end{abstract}

It has long been clear that spacing of explicit learning (distributing a fixed amount of study time for certain materials over a longer period) can powerfully increase the probability that these materials can be recalled. Less well known are the inconsistent effects of spacing on other kinds of learning, in particular those related to skill acquisition. In the present study, we examine the effects of temporal spacing on a particular form of skill learningthe performance improvement that occurs as people repeatedly do arithmetic calculations. This form of learning differs from that considered in the "standard" studies of spacing in the episodic memory literature in at least two respects. First, the information recalled is not taught to the learner by the experimenter, but is self-produced. Second, the most conspicuous change is in speed of response, rather than accuracy. For reasons we discuss, effects of spacing in this situation might not parallel those found with episodic memory designs.

\section{Spacing Effects}

Evidence that spacing can enhance recall probability goes back to Ebbinghaus (1885/1964). Spacing has been shown to be quite robust in a variety of tasks involving verbal episodic recall (see Cepeda, Pashler, Vul, Wixted, \& Rohrer, 2006 , for a recent review). Various studies have documented the fact that spacing increases the probability of success in cued recall and paired associate tasks with long retention intervals (e.g., Glenberg, 1976; Glenberg \& Lehmann, 1980; Pashler, Rohrer, Cepeda, \& Carpenter, 2007; Rumelhart, 1967). Spacing can also help children recall newly taught mathematical facts (Rea \& Modigliani, 1985).

However, when one looks across the broad category of "skill learning" or "implicit memory," tasks where the re- sponse does not typically involve explicit recollection, the beneficial effects of spacing are far less clear. For example, spacing effects do not seem to be robust for perceptual identification and word fragment completion tasks (Greene, 1990; Perruchet, 1989). In our lab, we did not find substantial spacing effects for tasks involving visuospatial categorization learning (Pashler et al., 2007).

\section{Transitions From Calculation to Retrieval}

A particularly prominent consequence of arithmetic skill learning is a gradual increase in the occurrence of direct memory retrieval — directly recollecting the answer in one step, rather than relying on calculation using an explicit algorithm. There has been debate about whether retrieval occurs simultaneously with calculation on any given trial (as suggested by Logan, 1988; Palmeri, 1997) or merely supplants calculation (Rickard, 1997, 2004). There is little doubt, however, that with repeated exposure to a given arithmetic problem, retrieval becomes more frequent.

\section{The Present Experiments}

The present study poses a fairly straightforward question that bridges the topics of spacing and the algorithmto-retrieval transition. We ask how spacing of training on specific problems affects this transition. Spacing of learning was varied within a session, by manipulating the "set size" of arithmetic problems given during training (i.e., the number of problems performed before those problems were repeated). The larger the set, the greater the average temporal spacing between successive re-presentations of a problem. This variable strongly influences the learning of new associations (Pashler, Zarow, \& Triplett, 2003).

T. C. Rickard, trickard@ucsd.edu 
Several hypotheses present themselves. On the one hand, one might expect a spacing effect trade-off similar to those observed in the verbal recall literature, such that although spacing results in a slower rate of performance improvement during training, it improves performance on the test. Schmidt and Bjork (1992) suggest that this tradeoff is common, and they point to spacing as one prominent example of a variable that produces it.

On the other hand, given the tenuousness of spacing effects in implicit learning tasks generally, one could hypothesize that such effects would be weak or absent. The underlying learning system may be different from that engaged by explicit memory tasks and it may be subject to different temporal dynamics. There is a second reason to suppose that spacing might not benefit arithmetic skill learning. One might suppose that to learn the transition from calculation to retrieval, it is best to actually engage in retrieval (an example of the rule "To learn X, practice doing X"). If long spacing reduces the probability of using retrieval during training, one might expect it to reduce the learning of the retrieval pathway. This account would predict that shorter spacing is associated with faster performance and greater use of retrieval in both training and test sessions.

\section{EXPERIMENT 1}

Our task required subjects to multiply a single-digit number by a two-digit number (e.g., $6 \times 18$ ), a task that few adults can perform using the retrieval strategy without training. In Experiment 1, there were two sessions. In the training session, multiplication problems were presented, some with short interitem spacing and others with long interitem spacing. In the test session, problems were presented in random order.

\section{Method}

Subjects. Thirty-nine subjects from the University of California, San Diego participated for course credit. Ten subjects did not complete the experiment, leaving data from 29.

Materials. The experiment involved 24 multiplication problems (the problems are listed in the Appendix). Each problem required multiplying a two-digit number by a one-digit number.

Design. Session 1 involved training, and Session 2 involved a test. Every subject was taught all 24 problems within Session 1, receiving 15 exposures to each problem. Every subject practiced 12 of the problems in what is termed the set size twelve (SS12) condition; the other 12 were practiced in the set size three (SS3) condition.

For the SS12 condition, the computer presented all 12 problems in a random order, then presented the same 12 problems in a different random order, and so forth, until all 12 problems had been presented 15 times.

The 12 problems taught in the SS3 condition were split into four groups of 3 (randomly and individually for each subject). Each group was practiced 15 times without any other items intervening. The computer presented all 3 items from a group in a random order, then presented the same group in a new random order, and so forth until the group of 3 problems had been presented 15 times, for a total of 45 presentations. Then the computer moved on to the next group of 3 items, and so forth until all 12 items had been presented 15 times. In both conditions, the constraint was enforced that the same problem could never appear twice in succession (an event that might otherwise have occurred at the boundary between successive presentations of a set).
To ensure that set size was not confounded with item difficulty or position within the training period, subjects were randomly assigned to one of four counterbalancing conditions. These conditions determined which of two halves of the problem list were assigned to SS3 versus SS12 (Problem Groups A and B in the Appendix), and also determined whether the training on SS3 came before or after the training on SS12.

Procedure. Each subject was run individually in a moderately illuminated soundproof room. Subjects were told that they would be solving multiplication problems in their head, without pen and paper. More specifically, they were instructed to do these problems in a standard way-by multiplying the single-digit number with the tens place of the double-digit number, then multiplying the singledigit number by the ones place of the double-digit number, and then adding the two products. Subjects were asked to say the answer aloud as soon as they thought they knew it. After the computer picked up the voice, the answer to the problem appeared on the screen. The experimenter pressed one of three buttons to indicate whether the response was correct or incorrect, or that there had been a malfunction (e.g., the voice key tripping off of a subject's cough or throat clearing, etc.). During both the training and the test sessions, if the subject's response was wrong, the correct answer was presented for $1 \mathrm{sec}$, after a delay of $1 \mathrm{sec}$. The next trial began after a further 1 -sec delay. There was a 1-min pause between the first and second halves of the task.

Session 2 occurred 7 days after Session 1 . The procedure was as follows: The subject was presented with all 24 problems in a random order, then the same 24 problems were presented in a new random order, and so on for eight runs through the list. Thus, the session consisted of 192 problems, half of which were taught in SS3 and half of which were taught in SS12. The problems were presented without rest breaks.

\section{Results and Discussion}

Figure 1 shows the mean reaction times (RTs) for correct trials as a function of condition, session, and block number, where a block is a sequence of one presentation of each item in the set. As expected, the figure shows a steady decrease in RT over training. The decrease in RT was substantially greater, however, for SS3. This pattern was reversed on the test, where SS12 shows substantially enhanced performance in comparison with SS3. This crossover interaction (SS3 being faster in training, slower on test) was confirmed by a within-subjects ANOVA with a 2 (condition) $\times 2$ (session) factorial design $[F(1,28)=$ $70.6, p<.0001]$.

Error results were analogous. In the training session, mean error rates were .055 and .096 for SS3 and SS12, respectively. In the test session, the pattern reversed: The error rate was .081 for SS3, and .064 for SS12.

The results are clearly in line with the view suggested by Schmidt and Bjork (1992). Larger set sizes (greater spacing) reduce the rate of performance improvement during training but improve performance on the delayed test.

\section{EXPERIMENT 2}

Although the results of Experiment 1 provide a fine example of the generalization suggested by Schmidt and Bjork (1992), they do not provide any clear information on how spacing may have modulated the transition from calculation to retrieval. Drawing on the prior literature (e.g., Rickard, 2004), we hypothesized that the patterns 


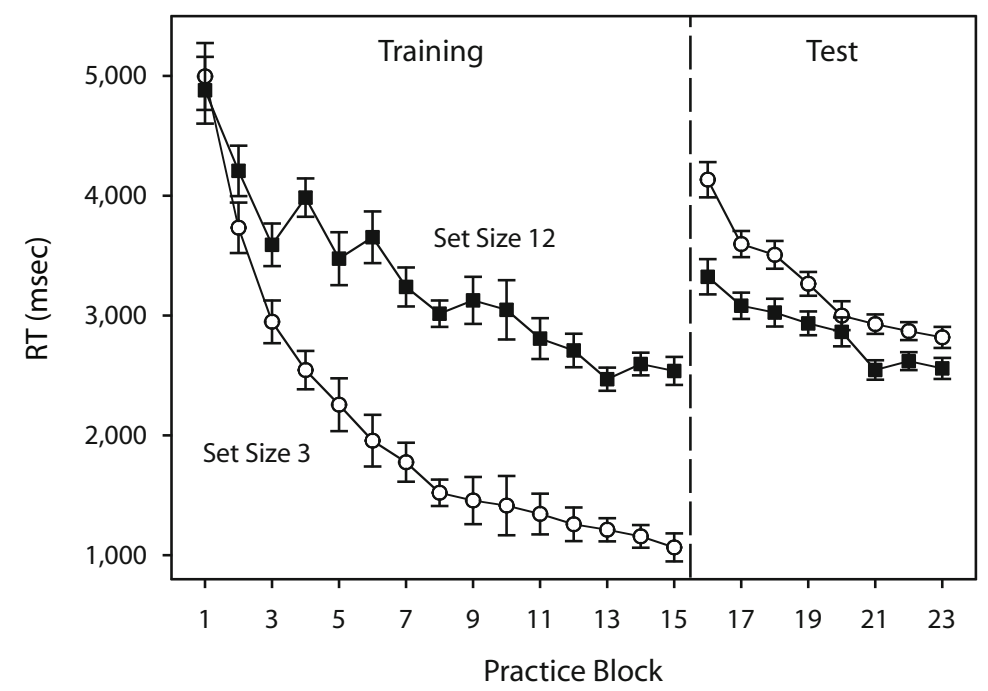

Figure 1. Mean reaction time (RT) as a function of set size, session, and block for Experiment 1. The vertical dashed line separates the training and test sessions. Error bars are standard errors corresponding to matched $t$ tests performed separately for each block.

in Experiment 1 reflected, to a large extent, different patterns of shift to retrieval for the two conditions. For the SS3 condition, the shift might have happened relatively quickly during practice. It appears, however, that the shift did not reflect stable long-term learning, and therefore that subjects often reverted to the slower algorithm strategy during the test. In the SS12 condition, the reverse appears to have happened. The transition to retrieval might have occurred for a smaller percentage of problems during training in that condition, but for the shifts that did occur there might have been more stable long-term learning. Hence, on the test, a higher percentage of retrievals occurred for problems in that condition. Experiment 2 was designed to test this account of the crossover interaction by using strategy probing.

\section{Method \\ Subjects. A total of 22 subjects participated in a three-session experiment. Of these, 21 were paid to participate, and 1 participated in the first two sessions in return for course credits and was paid for the last one. \\ Materials, Design, and Procedure. These aspects of Experi- ment 2 were identical to those of Experiment 1, except as noted here. Two training sessions (Sessions 1 and 2) were separated by a 2-day interval. On every trial of the last five blocks of Session 2, the subject was asked to indicate whether the answer had been reached by calculating, by retrieving from memory, or by using other means. The same strategy probing was also done on every trial of the test session. To indicate their strategy choice, subjects pressed one of three buttons on the button box. The wording was as follows: How did you arrive at your answer? Please press $C$ for "Calculation," $D$ for "Direct Retrieval," or O for "Other."}

\section{Results and Discussion}

Figure 2 shows the mean RTs for correct trials as a function of condition, session, and block number. The results for Session 1 mirror those of Experiment 1. On the first block of Session 2, there was a temporary reversal, such that subjects responded significantly faster in the SS12 condition than in the SS3 condition (3,632 vs. 2,989 msec) $[t(21)=3.78, p<.01] . \mathrm{SS} 3 \mathrm{RT}$ s were faster throughout the remainder of Session 2. Throughout the test session, subjects performed better on the problems trained in the SS12 condition, just as in Experiment 1.

The error rates mirrored the RTs. For SS3 problems, error rates were .057 and .020 for the first and second sessions, respectively, and .076 on the test. For SS12, the same values were $.095, .050$, and .069 .

The strategy probing results for the last five blocks of Session 2 and for the test session are shown in Figure 3. In Session 2, subjects were generally relying on direct retrieval in the SS3 condition but were doing so only about half the time in the SS12 condition. This pattern reversed in the test session, with direct retrieval reported more frequently for the SS12 problems.

To explore the possibility that the superior performance on the test in the SS12 condition was driven primarily or solely by the increased rate of retrieval in that condition, we computed mean RTs on the test for each condition and separately by strategy report ("algorithm" or "retrieval"; the relatively small number of "other" reports were excluded). Five subjects who did not report using both strategies in both conditions were excluded from this analysis. The overall analysis for this subset of subjects (collapsing over strategy) confirmed the advantage for SS12 that was reported in the analyses of the full set of subjects (means of 2,384 and 2,772 msec for SS12 and SS3, respectively) $[t(1,16)=3.27, p<.01]$.

RTs as a function of strategy and set size are shown in Figure 4. A 2 (strategy) $\times 2$ (condition) within-subjects ANOVA confirmed the strong effect of strategy $[F(1,16)=$ $51.8, p<.001]$, but there was no longer a significant effect of condition $[F(1,16)=2.06, p=.17]$, and there was no strategy $\times$ condition interaction $[F(1,16)=1.42, p=$ 


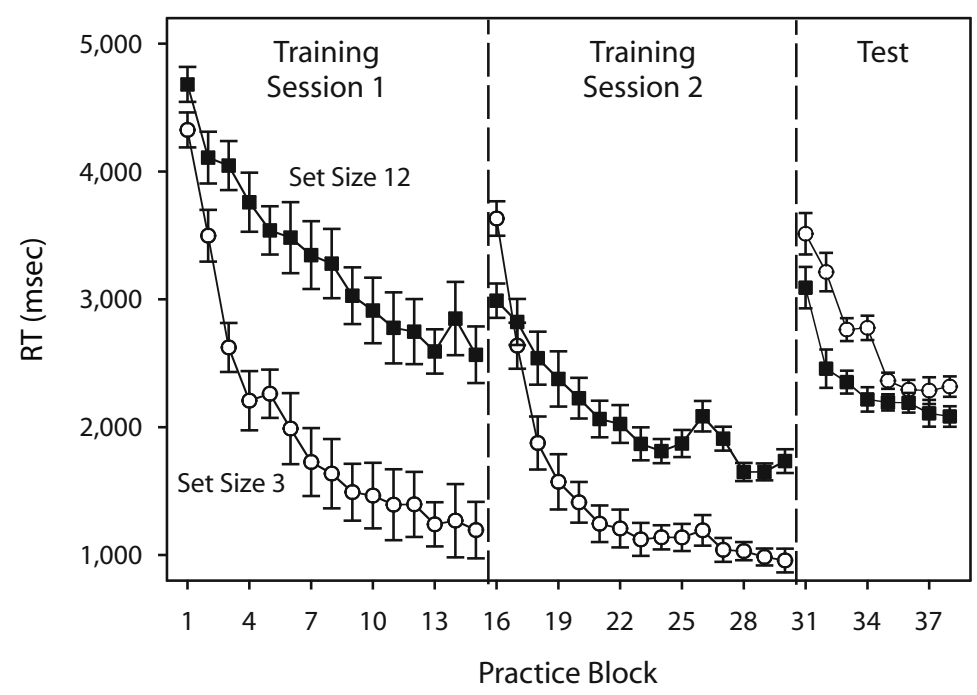

Figure 2. Mean reaction time (RT) as a function of set size, session, and block for Experiment 2. The vertical dashed lines separate the training and test sessions. Error bars are standard errors corresponding to matched $t$ tests performed separately for each block.

.25]. These results indicate that the condition difference in the overall test analysis was driven primarily by the increased use of the retrieval strategy in SS12.

Although these strategy reports are correlated with RT, several factors suggest that they provide a generally valid index of actual strategy use. First, on transfer tests, subjects report reverting to algorithm usage for new problems while continuing to use retrieval for old (previously practiced) problems (Rickard, 1997), showing that subjects do not simply report more retrieval use over the course of practice as a habit or to satisfy perceived demand characteristics (see also Rickard, 2004). Second, arithmetic algorithms are believed to involve subvocal intermediate steps, whereas direct retrieval does not. The distinction between algorithm and retrieval is thus an exemplary case in which subjects are expected to have access to memories for their mental processes when probed that are diagnostic of strategy use (Ericsson \& Simon, 1993). Third, a result from the present experiments supports the validity of strategy probing. For Session 1 of Experiment 2, the mean RTs on the first training block, which reflect use of the algorithm strategy, were 4,600 msec for the first set of problems trained in the SS3 condition, and 4,299, 4,102, and $4,195 \mathrm{msec}$, for the second, third, and fourth sets trained, respectively. These results suggest some general improvement in algorithm efficiency between the first and third

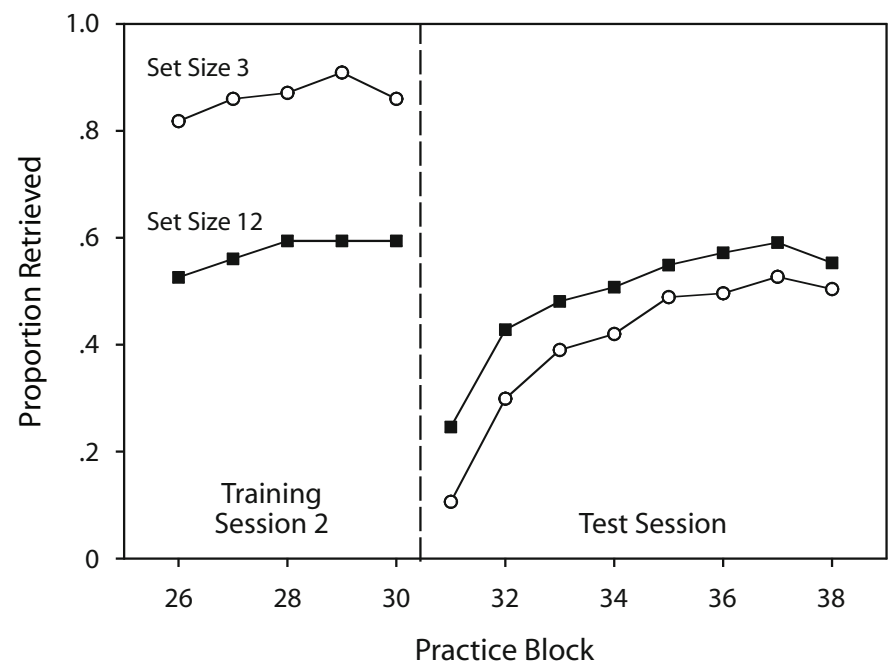

Figure 3. Proportion of direct retrieval reports as a function of set size, session, and block in Experiment 2. These data are from the last five blocks of the second training session and from the entire test session. The vertical dashed line separates the training and test sessions. 


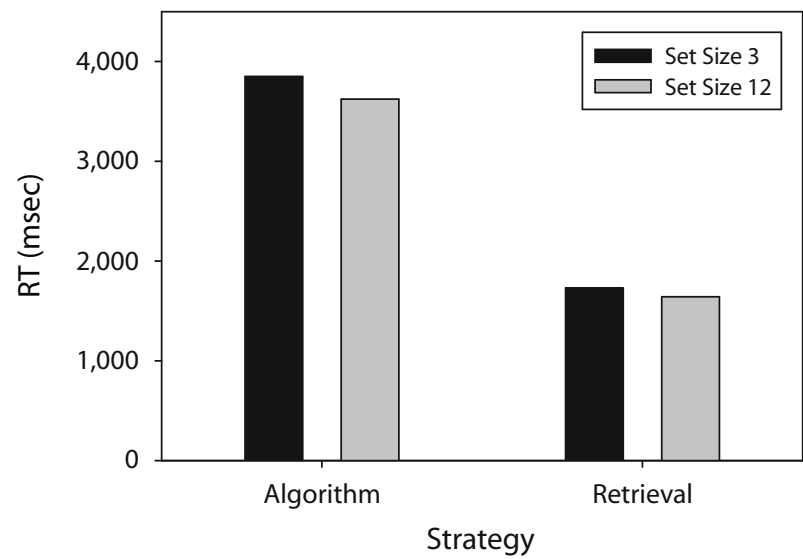

Figure 4. Mean reaction time (RT) as a function of set size and strategy in Session 3 of Experiment 2.

problem sets but not thereafter. Supporting validity of the algorithm reports, the means for the third and fourth sets are in the same range as the $3,851 \mathrm{msec}$ mean for the SS3 problems on the test when the algorithm was reported.

\section{GENERAL DISCUSSION}

In the introduction, it was pointed out that although spacing effects are ubiquitous in studies examining accuracy of recall for newly acquired associations, it is not so clear that spacing has a beneficial effect of the tuning that goes on during skill learning, which may rely more on "implicit memory" for which spacing effects seem not so robust (Greene, 1990; Perruchet, 1989). To explore this issue, we investigated whether greater spacing of arithmetic problems would promote or impede the speedup that comes with repetition training. The results showed that during training, greater spacing resulted in slower response latencies, less accuracy, and a much smaller likelihood of switching from calculation to memory retrieval. However, in the test sessions (in which time gaps between reoccurrences of any given problem were equated across condition) as well as on the first block of the second training session in Experiment 2, these effects were reversed. These results show that it is probably not best to arrange training in a way that maximizes the frequency of direct retrieval within a session (or that simply optimizes performance).

The results fit well with the general observation of Schmidt and Bjork (1992) that manipulations facilitating performance during training often reduce the degree or quality of learning. At a practical level, the results imply that spacing should probably be incorporated into drilling on arithmetic facts, even though this produces less apparent fluency during training.

Like many (but not all) manipulations of spacing, the present set size manipulation may have affected not only the time elapsed since previous encounters with a given problem, but also the likelihood that a problem was still represented in working memory. It may be the case, as some models of spacing have suggested (Young, 1966), that associations are strengthened more when the an- swer is retrieved from long-term memory rather than from working memory. In principle, it would be possible to manipulate both timing and the number of intervening problems separately, and thus determine which of these variables is most responsible for the effects observed here.

Another well-known account of spacing attributes the effect to changes in the context that is present at the time of encoding. As spacing between repetitions is increased, there is more time for the encoding context to have drifted, resulting in a greater expected difference between contexts. On certain assumptions, this could make it more likely that the context at test is similar to the context present during at least one of the encoding events (Glenberg, 1979; Howard \& Kahana, 2002; Whitten \& Bjork, 1977). This account of the present results might seem viable. However, encoding variability models have difficulty accounting for certain findings in the literature (e.g., Ross \& Landauer, 1978).

The present results make it clear that robust spacing effects can occur in skill-learning situations in which latency is the critical variable. Thus, the boundary between the many situations in which spacing effects are found and those in which they are not (several of which are described in the introduction to this article) is one that needs to be charted in future research. Characterizing this boundary should be important for translational applications of learning science and may provide new insights into the distinctions among underlying memory systems.

\section{AUTHOR NOTE}

This work was supported by the Institute of Education Sciences (U.S. Department of Education Grants R305H040108 and R305H040108 to H.P.) and National Science Foundation (Grant BCS-0720375, H.P., PI; and Grant SBE-0542013, G. W. Cottrell, PI). David Cun provided expert programming assistance in this project. Correspondence concerning this article should be addressed to T. C. Rickard, Department of Psychology, 0109, University of California, San Diego, 9500 Gilman Drive, La Jolla, CA 92093-0109 (e-mail: trickard@ucsd.edu).

\section{REFERENCES}

Cepeda, N. J., Pashler, H., Vul, E., Wixted, J. T., \& Rohrer, D. (2006). Distributed practice in verbal recall tasks: A review and quantitative synthesis. Psychological Bulletin, 132, 354-380.

Ebbinghaus, H. (1964). Memory: A contribution to experimental psychology (H. A. Ruger \& C. E. Bussenius, Trans.). New York: Dover. (Original work published 1885)

Ericsson, K. A., \& Simon, H. A. (1993). Protocol analysis: Verbal reports as data. Cambridge, MA: MIT Press.

Glenberg, A. M. (1976). Monotonic and nonmonotonic lag effects in paired-associate and recognition memory paradigms. Journal of Verbal Learning \& Verbal Behavior, 15, 1-16.

Glenberg, A. M. (1979). Component-levels theory of the effects of spacing of repetitions on recall and recognition. Memory \& Cognition, 7, 95-112.

Glenberg, A. M., \& Lehmann, T. S. (1980). Spacing repetitions over 1 week. Memory \& Cognition, 8, 528-538.

GreENE, R. L. (1990). Spacing effects on implicit memory tests. Journal of Experimental Psychology: Learning, Memory, \& Cognition, 16, 1004-1011.

Howard, M. W., \& KaHANA, M. J. (2002). A distributed representation of temporal context. Journal of Mathematical Psychology, 46, 269-299.

Logan, G. D. (1988). Toward an instance theory of automatization. Psychological Review, 95, 492-527.

PAlmeri, T. J. (1997). Exemplar similarity and the development of au- 
tomaticity. Journal of Experimental Psychology: Learning, Memory, \& Cognition, 23, 324-354.

Pashler, H., Rohrer, D., Cepeda, N. J., \& Carpenter, S. K. (2007). Enhancing learning and retarding forgetting: Choices and consequences. Psychonomic Bulletin \& Review, 14, 187-193.

Pashler, H., Zarow, G., \& Triplett, B. (2003). Is temporal spacing of tests helpful even when it inflates error rates? Journal of Experimental Psychology: Learning, Memory, \& Cognition, 29, 1051-1057.

PERrUChet, P. (1989). The effect of spaced practice on explicit and implicit memory. British Journal of Psychology, 80, 113-130.

Rea, C. P., \& Modigliani, V. (1985). The effect of expanded versus massed practice on the retention of multiplication facts and spelling lists. Human Learning: Journal of Practical Research \& Applications, 4, 11-18.

RICKARD, T. C. (1997). Bending the power law: A CMPL theory of strategy shifts and the automatization of cognitive skills. Journal of Experimental Psychology: General, 126, 288-311.

RICKARD, T. C. (2004). Strategy execution in cognitive skill learning: An item-level test of candidate models. Journal of Experimental Psychology: Learning, Memory, \& Cognition, 30, 65-82.

Ross, B. H., \& Landauer, T. K. (1978). Memory for at least one of two items: Test and failure of several theories of spacing effects. Journal of Verbal Learning \& Verbal Behavior, 17, 669-680.

RUMELhaRT, D. E. (1967). The effects of interpresentation intervals on performance in a continuous paired-associate task (Tech. Rep. No. 116). Stanford, CA: Stanford University Institute for Mathematical Studies in the Social Sciences.

SCHMIDT, R. A., \& BJORK, R. A. (1992). New conceptualizations of practice: Common principles in three paradigms suggest new concepts for training. Psychological Science, 3, 207-217.

Whitten, W. B., II, \& BJoRK, R. A. (1977). Learning from tests: Effects of spacing. Journal of Verbal Learning \& Verbal Behavior, 16, 465-478.

YouNG, J. L. (1966). Effects of intervals between reinforcements and test trials in paired-associate learning (Doctoral dissertation, Stanford University, 1966). Dissertation Abstracts International, 27, 3699.

\section{APPENDIX}

\begin{tabular}{cc}
\hline Problem Group A & Problem Group B \\
\hline $2 \times 19$ & $2 \times 26$ \\
$2 \times 23$ & $3 \times 14$ \\
$3 \times 22$ & $3 \times 27$ \\
$4 \times 13$ & $4 \times 17$ \\
$4 \times 24$ & $5 \times 19$ \\
$5 \times 16$ & $5 \times 21$ \\
$6 \times 18$ & $6 \times 24$ \\
$6 \times 27$ & $7 \times 18$ \\
$7 \times 26$ & $7 \times 23$ \\
$8 \times 14$ & $8 \times 16$ \\
$8 \times 21$ & $9 \times 13$ \\
$9 \times 17$ & $9 \times 22$ \\
\hline
\end{tabular}

(Manuscript received September 21, 2007; revision accepted for publication November 13, 2007.) 